\title{
INFLUÊNCIA DE DIFERENTES SOLVENTES UTILIZADOS NA DEPOSIÇÃO DE FILME DE POLI(9-VINILCARBAZOL) EM DISPOSITIVOS OLEDS
}

\author{
Emerson Roberto Santos*,a, Shu Hui Wanga, Fabio Conte Correia ${ }^{a}$, Ivan Rodrigues Costa ${ }^{\mathrm{b}}$, Victor Sonnenberg ${ }^{\mathrm{b}}$, Elvo \\ Calixto Burini Juniorc e Roberto Koji Onmorid \\ aDepartamento de Engenharia Metalúrgica e de Materiais, Escola Politécnica da Universidade de São Paulo, 5508-900 São Paulo \\ - SP, Brasil

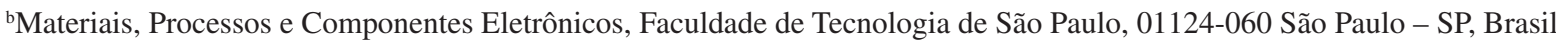 \\ 'Instituto de Energia e Ambiente da Universidade de São Paulo, 05508-010 São Paulo - SP, Brasil \\ ${ }^{\mathrm{d}}$ Departamento de Engenharia de Sistemas Eletrônicos, Escola Politécnica da Universidade de São Paulo, 05508-970 São Paulo \\ - SP, Brasil
}

Recebido em 24/01/2013; aceito em 23/07/2013; publicado na web em 21/08/2013

\begin{abstract}
INFLUENCE OF DIFFERENT SOLVENTS USED IN FILM DEPOSITION OF POLY(9-VINYLCARBAZOLE) IN OLED DEVICES. This work studies the influence of the film deposition process on light emission performance and on threshold voltage of OLEDs, with architecture glass/ITO/PEDOT:PSS/PVK/Alq $/$ /Al. The commercial PVK was dissolved in different solvents such as: chloroform, tetrahydrofuran, 1,2,4-trichlorobenzene and trimethylpentane. OLEDs were characterized by current-voltage and revealed a significant influence of the solvents, although all devices emitted green electroluminescence. A difference in threshold voltage up to $10 \mathrm{~V}$ was observed among OLEDs prepared from different solvents. The 1,2,4-trichlorobenzene showed best performance, presenting lowest treshold voltage $(\approx 6 \mathrm{~V})$, followed by tetrahydrofuran $(\approx 8 \mathrm{~V})$, trimethylpentane $(\approx 14 \mathrm{~V})$ and chloroform $(\approx 16 \mathrm{~V})$.
\end{abstract}

Keywords: organic solvent; PVK; OLED.

\section{INTRODUÇÃO}

O fenômeno de emissão de luz, também chamado de eletroluminescência, em dispositivos OLEDs (diodos orgânicos emissores de luz) foi observado pela primeira vez por volta de 1960, em uma experiência envolvendo campos elétricos em cristais de antraceno. ${ }^{1}$ Como esses dispositivos apresentavam elevadas tensões de limiar e baixa eficiência, a utilização de materiais orgânicos na fabricação de dispositivos foi praticamente descartada por, pelo menos, duas décadas. Após esse período, a utilização de diferentes estruturas orgânicas na fabricação dos dispositivos voltou a impulsionar as pesquisas relacionadas ao assunto, pois foi verificada uma melhoria na eficiência quando comparado com os dispositivos produzidos anteriormente, dado que a tensão de operação destes foi diminuída significativamente. ${ }^{2}$ Paralelamente ao avanço das pesquisas em dispositivos orgânicos ocorrera também o estudo das propriedades eletrônicas de polímeros conjugados, que data do final da década 70 , com o advento dos polímeros condutores. ${ }^{3}$ Nas décadas seguintes, a descoberta de novos polímeros com propriedades eletrônicas interessantes, sob o ponto de vista comercial, fez com que aumentasse o interesse pela aplicação destes materiais em dispositivos eletroluminescentes. No início da década de 90 surge o primeiro diodo emissor de luz com camada ativa completamente polimérica. Este fato mostrou de modo consolidado o caminho da pesquisa em dispositivos poliméricos. ${ }^{4}$

Atualmente, apesar dos avanços alcançados nesta área, alguns pontos específicos ainda não estão completamente esclarecidos, dentre os quais podem ser citados: o mecanismo de transporte e de injeção de portadores de carga elétrica, o processo de recombinação das cargas elétricas para a emissão de luz, os mecanismos de degradação e os fatores que influenciam na eficiência, bem como a

*e-mail: emerson@lme.usp.br influência do processamento de materiais utilizados na construção de um dispositivo OLED.

Portanto, o estudo das propriedades eletrônicas destes materiais pode resultar não somente em melhoria no desempenho, como também aprimorar novas configurações de dispositivos.

As publicações mais recentes sobre dispositivos OLEDs fornecem uma visão do impacto revolucionário desta tecnologia no mundo contemporâneo e, devido ao grande interesse por parte das indústrias multinacionais, o desenvolvimento dos OLEDs têm obtido amplo sucesso, motivo pelo qual estão sendo implementados atualmente em equipamentos de consumo..$^{5-7}$ Esse fato tem ocorrido devido às propriedades ópticas e elétricas interessantes alcançadas por esses dispositivos, sob o ponto de vista custo/benefício, além deles terem adquirido maior estabilidade ao longo desses últimos anos aumentando ainda mais a sua confiabilidade. Com o avanço dessa nova tecnologia, vários grupos de pesquisa na área acadêmica e industrial vêm trabalhando, visando o aprimoramento contínuo desses dispositivos para as mais diversas aplicações. ${ }^{8-11}$

Os dispositivos OLEDs possuem um processo de fabricação mais simplificado quando comparado aos LEDs inorgânicos tradicionais. O processo de fabricação dos OLEDs é baseado no empilhamento de diferentes tipos de camadas, cada uma com funcionalidade específica. ${ }^{12,13}$

A arquitetura explorada neste trabalho consiste na utilização dos seguintes materiais: (1) óxido transparente condutivo (TCO) depositado sobre a base do dispositivo, que é o vidro (mas que também poderia ser de plástico) transparente para a passagem da luz. Este TCO possui duas características distintas, como transparência e condutividade elétrica para promover a injeção de cargas elétricas do tipo buracos que são injetados na camada subsequente, ou seja, funcionando como eletrodo anodo; (2) sobre o TCO é depositada uma camada conhecida como HTL (camada transportadora de buracos), que tem a função de melhorar o transporte de portadores de cargas do tipo buracos, que serão injetados na camada ativa. Esta camada 
é constituída por um polímero semicondutor, que deve possuir características ópticas e elétricas similares aos do filme de TCO; (3) em seguida, é depositada a camada ativa do dispositivo OLED, que é constituída por um polímero ou material orgânico eletroluminescente e que geralmente é diluído em algum solvente orgânico. Esta camada recebe os portadores de carga elétrica (elétrons e buracos) que se recombinam, propiciando a emissão de luz; (4) sobre a camada ativa é depositada a ETL (camada transportadora de elétrons), que tem a função de melhorar o transporte de portadores de cargas do tipo elétrons que são injetados na camada ativa; (5) finalizando o empilhamento, é depositada uma camada metálica que funcionará como eletrodo catodo durante a polarização do dispositivo.

É importante ressaltar que também existem outras arquiteturas muito diferentes da explorada neste trabalho, mas que possuem o mesmo princípio de funcionamento. ${ }^{14-22}$ Este tipo de estrutura de dispositivo aqui apresentada é a que está sendo mais estudada no momento, porém, apenas o clorofórmio tem sido utilizado como solvente orgânico para diluição do material emissor.

\section{PARTE EXPERIMENTAL}

Foram utilizadas lâminas de vidro nas dimensões $25 \times 25 \mathrm{~mm}$ como substrato para as análises ópticas, elétricas e também para a fabricação dos dispositivos. As lâminas fornecidas pela empresa Displaytech apresentam camada de TCO constituída por ITO (óxido de índio e estanho) e com resistência de folha de $15 \Omega / \square$. Antes da deposição das camadas para a montagem dos dispositivos OLEDs as lâminas foram limpas com detergente doméstico e água corrente para a eliminação de pó e particulados. Em seguida, as amostras foram colocadas em um porta-amostra e submersas, primeiramente em álcool isopropílico e depois em acetona, por 30 minutos em cada solvente. Todo esse procedimento de limpeza foi realizado em banho ultrassônico. Após a limpeza, as lâminas foram submetidas ao tratamento por UV-Ozônio (em um aparato previamente montado) durante o tempo de 5 minutos, para melhorar a molhabilidade da superfície. ${ }^{23}$ Esse tratamento também ajuda na eliminação de contaminantes, tais como compostos carbonilados e hidrocarbonetos, que diminuem o desempenho final dos dispositivos. ${ }^{24}$ Imediatamente após o tratamento com UV-Ozônio, sobre os filmes de ITO foi depositado o polímero PEDOT:PSS [poli-(3,4-etilenodioxitiofeno):poli-(p-estirenosulfonato de sódio)] (dispersão aquosa a 1,3\% e fornecido pela empresa SigmaAldrich) utilizado como HTL. O polímero foi depositado pela técnica de spin-coating, a 1800 RPM por 35 segundos, utilizando um aparato montado a partir de um disco rígido desmontado de computador e, em seguida, as amostras foram deixadas em estufa a $80^{\circ} \mathrm{C}$ durante 20 minutos, para a evaporação do solvente (água). ${ }^{25,26}$

O polímero emissor poli(9-vinilcarbazol) (PVK) foi fornecido pela Sigma-Aldrich e as soluções com este polímero foram preparadas previamente. O PVK foi dissolvido em diferentes solventes orgânicos como: 1,2,4-triclorobenzeno, tetrahidrofurano, trimetilpentano e clorofórmio, resultando em soluções com a concentração estabelecida em $10 \mathrm{mg} / \mathrm{ml}$. As soluções também foram depositadas pela técnica de spin-coating formando filmes finos de PVK diretamente sobre a camada de PEDOT:PSS. Os parâmetros de processo de deposição utilizados foram os mesmos anteriormente descritos e a secagem em estufa foi efetuada em seguida a $50{ }^{\circ} \mathrm{C}$ por 60 minutos. ${ }^{27}$ Após a evaporação, as amostras foram carregadas em uma evaporadora térmica a vácuo com duas fontes de evaporação distintas. Em uma das fontes foi colocado um cadinho contendo o material ETL, conhecido como $\mathrm{Alq}_{3}$ (tris(8-hidroxiquinolina) alumínio) sintetizado em nosso laboratório, o qual foi evaporado e depositado primeiramente sobre a camada de PVK. Na outra fonte foi colocado outro cadinho com filetes de alumínio, que foi evaporado sobre a camada de $\mathrm{Alq}_{3}$ formando o eletrodo catodo dos dispositivos. Terminado esse procedimento, os dispositivos foram levados para as caracterizações elétrica e óptica.

Neste trabalho também foram preparadas 2 amostras para caracterização de resistência elétrica dos filmes de ITO/vidro, 2 amostras para caracterização de resistência elétrica dos filmes de PEDOT:PSS depositado sobre ITO/vidro, 8 amostras (4 para caracterização de resistência elétrica e 4 amostras para caracterização óptica) dos filmes de PVK depositados sobre a estrutura PEDOT:PSS/ITO/vidro.

A medição da resistência elétrica dos filmes foi realizada com a utilização de um multímetro digital, fabricado pela Minipa, modelo 2082A. A amostra em análise foi colocada sobre um padrão (com tamanho idêntico ao da amostra $25 \times 25 \mathrm{~mm}$ ) impresso em folha de papel sulfite, com 18 pares de pontos no total, sendo distribuídos em três colunas. A medição da resistência elétrica foi realizada para cada par de pontos. A distância entre cada par de pontos foi mantida em $3 \mathrm{~mm}$, sendo que todos os pares de pontos foram distribuídos por toda a amostra.

Para a obtenção das curvas de corrente-tensão (I x V) dos dispositivos, foi utilizada uma fonte de tensão digital fabricada por encomenda (http://www.faiscas.net/). Durante a polarização dos dispositivos, utilizamos o software hyper terminal (incluso no sistema operacional Windows XP). Neste software foram pré-estabelecidos alguns parâmetros para as medidas, tais como: corrente elétrica máxima de $100 \mathrm{~mA}$, tensão máxima de $25 \mathrm{~V}$ e intervalo de incremento de tensão em $0,25 \mathrm{~V}$.

Para as análises ópticas dos filmes de PVK utilizamos um microscópio da marca Olympus, modelo BX60M, com uma câmera acoplada ao equipamento. Nesta análise foram observadas as superfícies dos diferentes filmes de PVK depositados sobre a estrutura PEDOT:PSS/ITO/vidro. Obtivemos imagens com ampliações de: 5, 10, 20 e 50 vezes dos filmes de PVK, obtidos a partir das soluções com os diferentes solventes.

Para completar as análises ópticas dos filmes de PVK (também depositados sobre a arquitetura PEDOT:PSS/ITO/vidro) foram realizadas medidas de transmitância. Nesta técnica, utilizamos o espectrofotômetro da marca Shimadzu, modelo UV-1650 PC, fazendo uma varredura no comprimento de onda de 350 a $1000 \mathrm{~nm}$.

Para obtenção dos espectros de emissão dos dispositivos utilizamos um espectrorradiômetro da marca Luzchem, modelo SPR-03, sendo que os dispositivos foram polarizados com 4,5 V utilizando a mesma fonte de energia. No espectrorradiômetro conectamos um cabo de fibra óptica, cuja extremidade foi colocada diretamente sobre a região ativa do dispositivo OLED. Com auxílio do software do próprio equipamento foram obtidos os espectros de emissão.

\section{RESULTADOS E DISCUSSÃO}

Após a limpeza química com solventes e também com tratamento superficial dos filmes de ITO com UV-Ozônio, selecionamos duas amostras de ITO/vidro e medimos a resistência elétrica nos 18 pares de pontos diferentes estabelecidos. Este mesmo método também fizemos para duas amostras de PEDOT:PSS depositados sobre a estrutura ITO/ vidro. A Figura 1 mostra a comparação entre os valores médios de resistência elétrica dos filmes de ITO/vidro e PEDOT:PSS/ITO/vidro com seus respectivos desvios padrões, sendo que as amostras analisadas com ITO/vidro foram nomeadas pelas letras A e B e as amostras com PEDOT:PSS/ITO/vidro foram nomeadas pelas letras C e D.

Com base nos resultados da Figura 1 ressalta-se a confiabilidade da metodologia empregada, que permitiu demonstrar a existência de pequenas diferenças na mesma amostra, bem como entre duas lâminas distintas.

Filmes de ITO fornecidos por outros fabricantes (Innovative Specialty Films e Delta Technologies) foram caracterizados no 


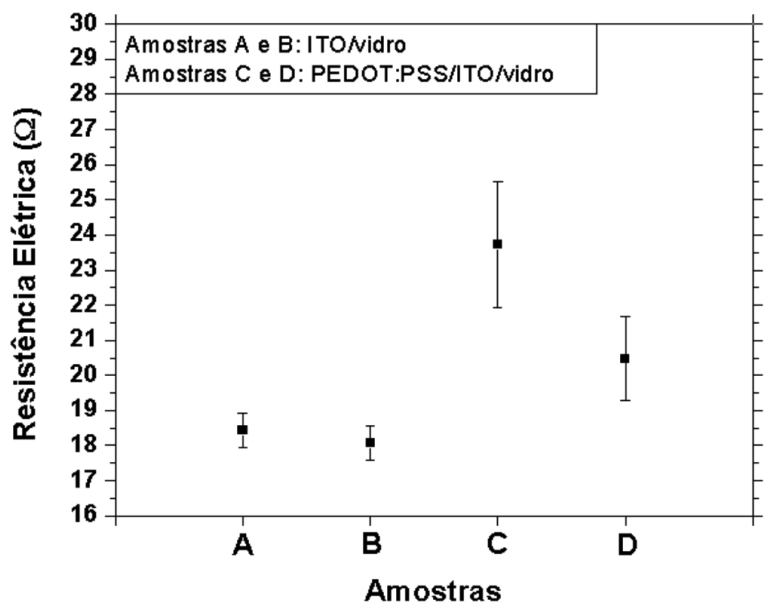

Figura 1. Resistência elétrica para duas amostras: filmes de ITO sobre vidro e filmes de PEDOT:PSS sobre ITO/vidro

trabalho de Satoru Yoshida utilizando o mesmo método, no qual foi verificado que as resistências elétricas também apresentaram pequenas diferenças. ${ }^{28}$ Ainda neste mesmo trabalho, mediu-se também a resistência de folha ao invés de resistência elétrica, com um equipamento Veeco, modelo FPP-100, e também foi encontrada uma pequena variação. Como as distâncias entre as pontas de prova foram mantidas constantes durante a varredura das medições no filme todo, a pequena variação encontrada nos valores de resistência elétrica pode estar relacionada com a falta de uniformidade dos filmes, uma vez que a condutividade elétrica depende da espessura do filme. ${ }^{29}$

Comparando-se as amostras de ITO/vidro com as amostras de PEDOT:PSS/ITO/vidro verificamos a ocorrência de uma resistência menor nos filmes de ITO. ${ }^{30}$

Utilizando a mesma metodologia de medição da resistência elétrica nos os filmes de PVK sobre a estrutura PEDOT:PSS/ITO/vidro, verificamos que os filmes de PVK também apresentaram variações nos valores de resistências elétricas em função de cada solvente orgânico. A Figura 2 mostra comparativamente os valores médios de resistência elétrica com seus respectivos desvios padrões para os filmes de PVK em função de cada solvente orgânico utilizado.

Com base nos resultados apresentados na Figura 2, verificamos o menor valor de resistência elétrica para os filmes de PVK obtidos com a solução de 1,2,4-triclorobenzeno, enquanto que o uso de tetrahidrofurano proporcionou maior resistência elétrica. Os valores de resistência elétrica encontrados estão muito próximos das grandezas obtidas pelos filmes de PEDOT:PSS depositados sobre ITO/vidro (Figura 1) e, com isso, fica evidente que os filmes de PEDOT:PSS e PVK formados apresentam condutividades elétricas similares, embora seja também conhecido o fato de que ambos os materiais funcionam como camadas transportadoras de buracos (HTL). ${ }^{31,32}$ A energia do HOMO do PVK é 5,8 eV, enquanto que o PEDOT:PSS apresenta uma função trabalho de 5,1 eV, ou seja, existe uma barreira de energia de $0,7 \mathrm{eV}$ comparativamente entre as duas camadas, ainda que tenham propriedades eletrônicas de transporte de buracos análogas. ${ }^{33,34} \mathrm{E}$ oportuno também mencionar que o recozimento do PEDOT:PSS em diferentes temperaturas mostrou a redução da resistência elétrica e o aumento da função trabalho, de modo que as propriedades elétricas dos polímeros são dependentes da sua morfologia. ${ }^{35}$

Além das medições de resistência elétrica foram realizadas análises ópticas, para verificar a morfologia presente nos diferentes filmes de PVK depositados sobre os filmes de PEDOT:PSS/ITO/ vidro. A Figura 3 mostra as imagens de microscopia óptica com diferentes ampliações, obtidas dos quatro filmes de PVK diluídos nos diferentes solventes.

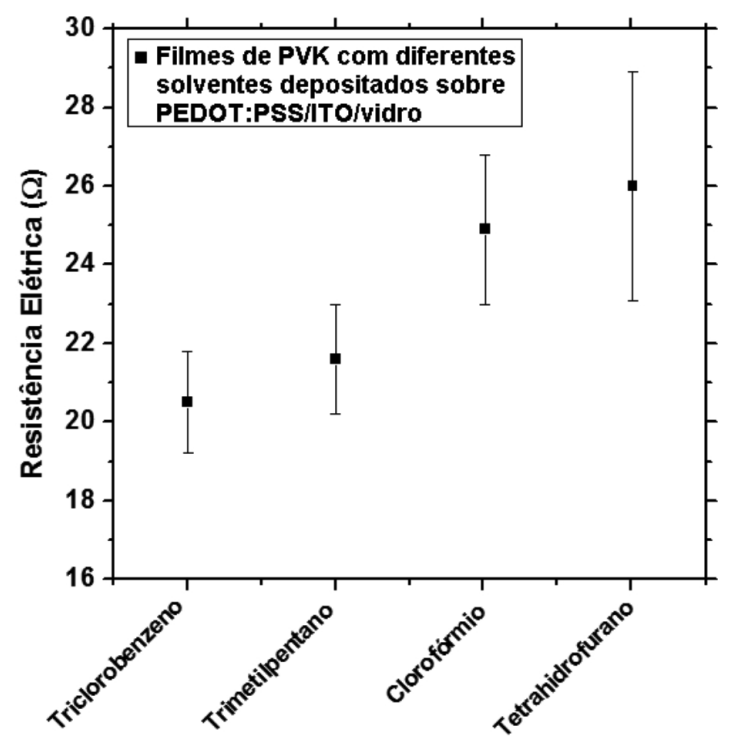

Figura 2. Resistência elétrica dos filmes de PVK com os diferentes solventes depositados sobre a estrutura PEDOT:PSS/ITO/vidro

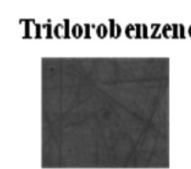

$5 \mathbf{x}$

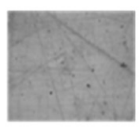

$10 x$

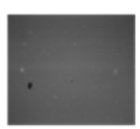

$20 \mathrm{x}$

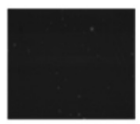

$50 x$

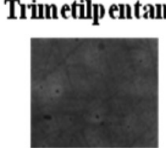

$5 \mathbf{x}$

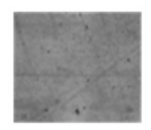

$10 x$

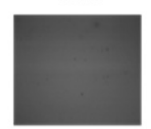

$20 x$

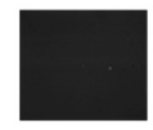

$50 x$

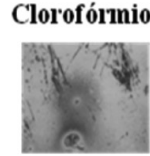

$5 x$

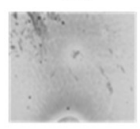

$10 x$

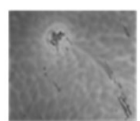

20x

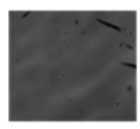

$50 x$

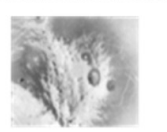

$5 x$

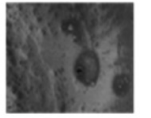

$10 \mathrm{x}$

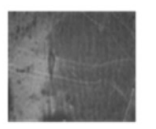

20x

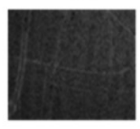

$50 x$
Tetrahidrofurano

Figura 3. Imagens obtidas por microscopia óptica dos filmes de PVK dissolvidos em diferentes solventes orgânicos

De acordo com as imagens obtidas dos diferentes filmes de PVK na Figura 3, verificamos maior uniformidade com a utilização do 1,2,4-triclorobenzeno e menor para o tetrahidrofurano, sendo que essa observação ficou mais evidente nas imagens com maior campo de visualização (ampliadas em 5 vezes). Estes resultados corroboram com aqueles encontrados na resistência elétrica (Figura 2) dos filmes de PVK, pois a maior uniformidade e menor resistência elétrica foram obtidas com os filmes utilizando-se 1,2,4-triclorobenzeno, enquanto que a menor uniformidade e maior resistência elétrica foram obtidas com os filmes utilizando-se tetrahidrofurano. A morfologia da superfície do filme de PVK formado é fortemente dependente da taxa de evaporação do solvente e as propriedades elétricas têm relação com a afinidade entre o solvente orgânico e o PVK.

Nas análises de transmitância dos filmes de PVK, verificamos que estes apresentaram elevada transparência na faixa do comprimento de onda da luz visível, embora o ITO e o PEDOT:PSS (utilizados como substrato) não sejam completamente transparentes. A Figura 4 mostra os resultados de transmitância em função do comprimento de onda para os filmes de PVK depositados sobre PEDOT:PSS/ITO/ vidro, para todos os solventes analisados. 


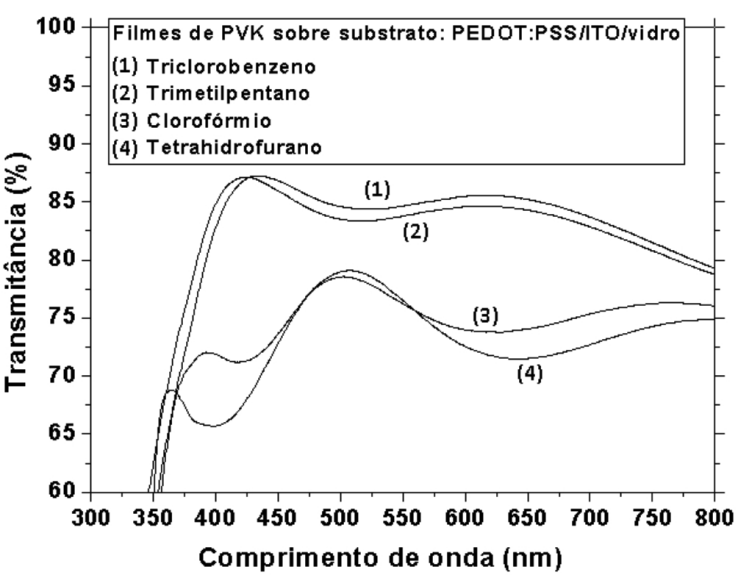

Figura 4. Comparação das transmitâncias dos filmes de PVK obtidos com os diferentes solventes, sobre o substrato de PEDOT:PSS/ITO/vidro

Os resultados mostraram maior transmitância de $\approx 85 \%$ para o filme obtido com 1,2,4-triclorobenzeno, seguido do trimetilpentano, ambos apresentando um pico maior de transmitância em $\approx 420 \mathrm{~nm}$. Os filmes com clorofórmio e tetrahidrofurano apresentaram valores de transmitância menores e com maior variabilidade, atingindo o ponto mais alto da transmitância em $\approx 75 \%$ para o comprimento de onda de $\approx 500 \mathrm{~nm}$. A faixa aproximada de 500 a $550 \mathrm{~nm}$ corresponde à cor verde no espectro eletromagnético, sendo que é nesta faixa que ocorre a eletroluminescência dos dispositivos. ${ }^{33}$

Com a mudança do solvente orgânico na deposição dos filmes de PVK verificamos que ocorreu uma variação no desempenho dos dispositivos e também comprometeu significantemente os comprimentos de onda da luz emitida pela eletroluminescência, incluindo um deslocamento pouco significativo no comprimento de onda do pico principal de emissão, como demonstramos na Figura (5).

Na Figura 5, as curvas dos OLEDs foram normalizadas no gráfico maior, enquanto que no gráfico menor foram mantidas as curvas conforme obtidas diretamente do software do equipamento. Dessa forma, os dispositivos com triclorobenzeno acabaram apresentando um comportamento muito parecido com os dispositivos montados com clorofórmio, seguido pelo comportamento do dispositivo montado com tetrahidrofurano, sendo que este apresentou maior quantidade de ruído. Os dispositivos montados com trimetilpentano apresentaram o maior ruído de todos e, devido a este comportamento, não foi colocado no gráfico. Se analisarmos as curvas de emissão, sob o ponto de vista mais qualitativo que quantitativo, verificamos que o gráfico menor situado na Figura 5 apresenta um comportamento muito diferente,

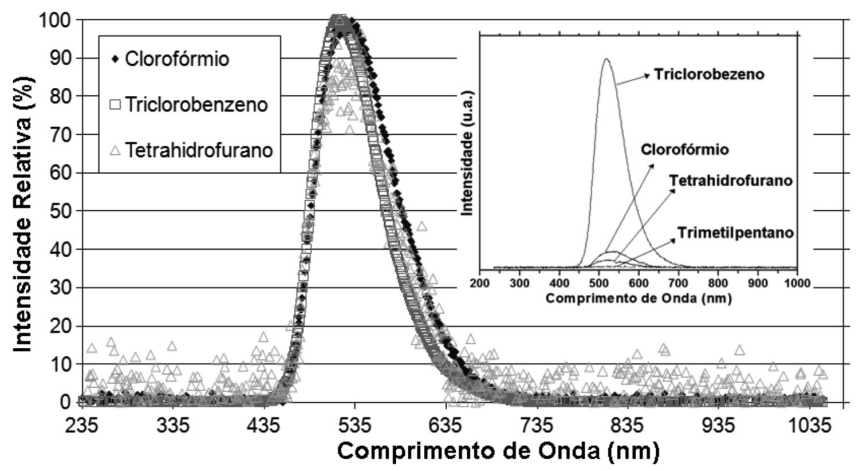

Figura 5. Intensidade espectral relativa da eletroluminescência obtida dos OLEDs montados com filmes de PVK diluído nos diferentes solventes. Nota: A relação sinal ruído da luminescência obtida para o dispositivo montado utilizando o solvente trimetilpentano é muito elevada e por esse motivo não foi colocada no gráfico levando-se em conta o cuidado de utilizarmos a mesma metodologia para extração dos resultados, o que coloca todos os dispositivos na mesma condição. Originalmente, o eixo das ordenadas (eixo y) é dado pelo software do espectrorradiômetro (Luzchem Research Inc. Spectroradiometer, versão 3.3) como intensidade, que é apenas uma forma representativa (e não absoluta) para comparação das intensidades alcançadas por cada OLED testado. Ainda nesta Figura 5, na qual consta o gráfico menor, verificamos que o dispositivo obtido com o uso de 1,2,4-triclorobenzeno apresentou um pico de emissão mais definido e intenso em $518 \mathrm{~nm}$, em comparação com os demais, o que corresponde à cor verde do espectro da luz visível. Para este pico de emissão, a tensão observada foi de $\approx 4,5 \mathrm{~V}$ e a corrente elétrica de aproximadamente $1 \mathrm{~mA}$. Dispositivos obtidos com a utilização de clorofórmio e tetrahidrofurano apresentaram picos de emissão muito menos intensos, obtidos nas mesmas condições de polarização, em comparação ao 1,2,4-triclorobenzeno. Em relação ao trimetilpentano verificamos que o espectro apresentou a intensidade muito reduzida em comparação com os outros dispositivos, porém, ampliando-se o espectro foi possível visualizar a emissão de luz na faixa de 500 a 550 nm, o que também corresponde à emissão de cor verde. A mudança do solvente utilizado na formação da camada ativa não deslocou significativamente os picos de emissão dos dispositivos e essa variação observada em todos os espectros não alterou significativamente a emissão de cor verde dos OLEDs.

É importante ressaltar que em outro estudo foi verificada a possibilidade da emissão de luz originar-se do $\mathrm{Alq}_{3}$ (utilizado como ETL), ao invés do PVK. Utilizando-se a mesma arquitetura, vidro/ITO/ PEDOT:PSS/PVK/ETL/Al, onde a camada de $\mathrm{Alq}_{3}$ foi substituída por Butyl-PBD, constatou-se a eletroluminescência na cor azul. ${ }^{35} \mathrm{O}$ fenômeno observado pode ser explicado pelo fato do PVK apresentar propriedades elétricas semicondutoras e contribuir na locomoção dos portadores do tipo buracos, levando ao incremento da recombinação de pares elétrons-buracos na camada injetora de elétrons $\left(\mathrm{Alq}_{3}\right)$, o que pode ter propiciado a emissão da luz verde, ao invés da recombinação de pares elétrons-buracos ocorrer no interior da camada de PVK.

Khalifa et al. montaram dispositivos com arquitetura vidro/ITO/ PEDOT:PSS/PVK/Al, ou seja, sem a camada $\mathrm{Alq}_{3}$ (como ETL) e não reportaram a eletroluminescência dos dispositivos, isso pode indicar mais uma evidência de que a emissão pode ser proveniente da camada de $\mathrm{Alq}_{3} \cdot{ }^{36}$

Para verificarmos o comportamento elétrico dos OLEDs montados com os diferentes filmes de PVK, os dispositivos foram polarizados nas mesmas condições. A Figura 6 mostra os resultados das curvas de corrente-tensão, obtidas para os diferentes dispositivos OLEDs montados.

É possível notar que o dispositivo com camada ativa de PVK utilizando 1,2,4-triclorobenzeno apresentou menor valor na tensão de limiar $(\approx 6 \mathrm{~V})$, enquanto que, utilizando o clorofórmio, obtivemos um valor mais elevado $(\approx 16 \mathrm{~V})$. Esse resultado revelou uma redução significativa da tensão de limiar apenas trocando o solvente orgânico.

Em trabalho anterior, utilizando a mesma arquitetura de dispositivo deste trabalho e clorofórmio como solvente do PVK, observou-se que os OLEDs apresentaram valores de tensão de limiar próximos ao encontrado neste trabalho. ${ }^{35}$

Uma hipótese que pode ser discutida em relação à tensão de limiar é a sua associação direta com a volatilidade dos solventes. Acredita-se que quanto menor a volatilidade do solvente orgânico, maior tempo haverá para as cadeias poliméricas se rearranjarem, melhorando e aumentando as interações intermoleculares e formando, portanto, uma estrutura mais contínua. Essa afirmativa corrobora o fato do 1,2,4-triclorobenzeno ser, dentre os escolhidos, o solvente com o maior ponto de ebulição $\left(213{ }^{\circ} \mathrm{C}\right)$, o que pode justificar a menor tensão obtida. Adicionalmente, o clorofórmio apresenta o menor 


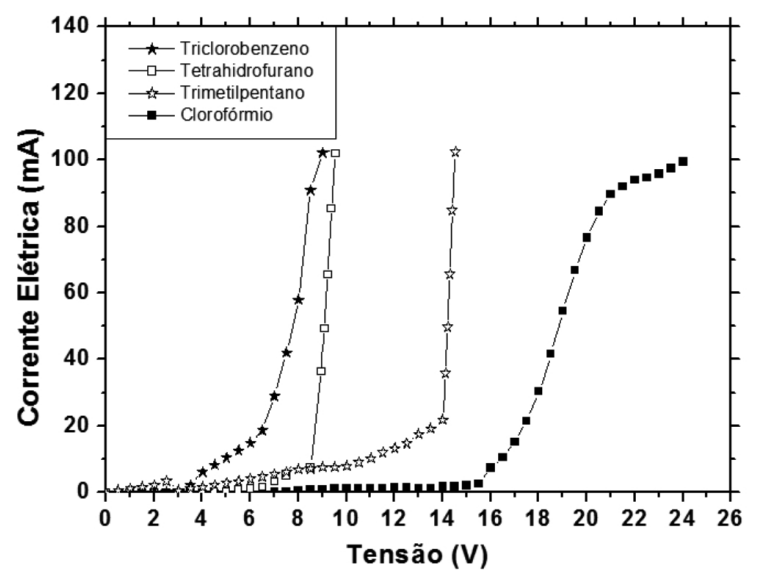

Figura 6. Curvas de corrente-tensão dos dispositivos OLEDs utilizando PVK diluído nos diferentes solventes

ponto de ebulição $\left(61{ }^{\circ} \mathrm{C}\right)$ proporcionando ao dispositivo OLED a maior tensão de limiar.

\section{CONCLUSÃO}

Filmes de PVK depositados sobre o mesmo substrato e obtidos a partir dos diferentes solventes, 1,2,4-triclorobenzeno, trimetilpentano, clorofórmio e tetrahidrofurano, apresentaram variações nos valores médios de resistência elétrica. A menor resistência elétrica foi observada com o uso do 1,2,4-triclorobenzeno e a maior com o tetrahidrofurano. Adicionalmente, a menor resistência elétrica correspondeu também à maior uniformidade dos filmes, observada por microscopia óptica. Nas caracterizações óptica e elétrica dos dispositivos foi possível verificar que o solvente orgânico altera significativamente a intensidade da eletroluminescência, provocando, inclusive, um deslocamento (porém, que foi pouco significativo) no comprimento de onda do máximo da banda de emissão principal. Neste trabalho, o 1,2,4-triclorobezeno foi o solvente que mais se destacou, pois, além de propiciar um aumento considerável na emissão de luz, ele também diminuiu consideravelmente a tensão de limiar para uma faixa de $\approx 10 \mathrm{~V}$. Os resultados observados indicam que a volatilidade do solvente durante a deposição do filme é possivelmente um parâmetro importante. Quanto maior o ponto de ebulição, mais lenta será a evaporação do solvente, o que aumenta o tempo de rearranjo das cadeias poliméricas para obtenção de um maior empacotamento, diminuindo a resistência elétrica do material e melhorando, portanto, a mobilidade dos portadores de carga.

\section{REFERÊNCIAS}

1. Pope, M.; Magnante, P.; Kallmann, H. P.; J. Chem. Phys. 1963, 38, 2042.

2. Tang, C. W.; Vanslyke, S. A.; Appl. Phys. Lett. 1987, 51, 913.

3. Chiang, C. K.; Fincher, C. R.; Jr.; Park, Y. W.; Heeger, A. J.; Shirakawa, H.; Louis, E. J.; Gau, S. C.; MacDiarmid, A. G.; Phys. Rev. Lett. 1977, 39, 1098

4. Burroughes, J. H.; Bradley, D. D. C.; Brown, A. R.; Marks, R. N.; Mackay, Y.; Friend, R. H.; Burns, P. L.; Holmes, A. B.; Nature 1990 347, 539.

5. Hurk, P. V. D.; Philips Research Password 2009, 34, 18.

6. Alessi, J. P.; Cottone, P. L.; 9th Congress of the International Colour Association, SPIE Dig. Lab. 2002, 4421, 947.

7. Chesterfield, R.; Johnson, A.; Lang, C.; Stainer, M.; Ziebarth, J.; Information Display Magazine 2011, 24.

8. Komoda T.; Panasonic Corporation, Core Technologies Development Center, Eco Solutions Company -2012; http://www.semiconwest.org/
sites/semiconwest.org/files/docs/Takuya\%20Komoda_Panasonic.pdf, acessada em Julho 2013.

9. Lee, K.-H.; Choi, H.-W.; Kim, K.-H.; Bark, C.-W.; Mol. Cryst. Liq. Cryst. 2012, 567, 78.

10. Lee, H. J.; Lee, E. J.; Lim, H. B.; Lee, J. M.; Jang, J.; Park, L. S.; Mol. Cryst. Liq. Cryst. 2012, 567, 132.

11. Colegrove, J.; Diplaysearch; Resumos do 12 th. Annual OLEDs World, San Francisco, EUA, 2010.

12. Santos, G.; Fonseca, F. J.; Andrade, A. M.; Deichmann, V.; Akcelrud, L.; Braga, S. S.; Coelho, A. C.; Gonçalves, I. S.; Peres, M.; Simões, W.; Monteiro, T.; Pereira, L.; J. Non-Cryst. Solids 2008, 354, 2897.

13. Braga, S. S.; Coelho, A. C.; Gonçalves, I. S.; Santos, G.; Fonseca, F. J.; Andrade, A. M.; Peres, M.; Simões, W.; Monteiro, T.; Pereira, L.; J. Non-Cryst. Solids 2008, 354, 2736.

14. Qiang, W. Z.; Jun, Z. C.; Heng, L. Mei, O. X.; Hong, Z. X.; Sci. China Chem. 2011, 54, 666

15. Predeep, P.; Hameed, T. A. S.; Aneesh, J.; Baiju, M. R.; Solid State Phenomena 2011, 171, 39.

16. Stewart, J. R. H. S.; Mattle, T.; Lippert, T. K.; Nagel, M.; Nuesch, F. A.; Wokaun, A.; J. Appl. Phys. 2013, 113, 043104-1.

17. Yang, X.; Yao, C.; Zhou G.; Platinum Met. Rev. 2013, 57, 2.

18. Lee, H. J.; Lee, E. J.; Lim, H. B.; Lee, J. M.; Jang, J.; Park, L. S.; Molecular Crystals and Liquid Crystals 2012, 567, 132.

19. Hyun, W. J.; Im, S. H.; Park, O. O.; Chin, B. D.; Journal of Information Display 2012, 13, 151.

20. Utochnikova, V. V.; Kotova, O.V.; Vaschenko, A. A.; Lepnev, L. S.; G., Vitukhnovsky, A. G.; Kuzmina, N. P.; Adv. Mater. Sci. Eng. 2012, $2012,9$.

21. Santos, E. R.; Correia, F. C.; Onmori, R. K.; Fonseca, F. J.; De Andrade, A. M.; Wang, S. H.; ECS Trans. 2012, 49, 347.

22. Santos, E. R.; Junior, E. C. B.; Hui, W. S.; Fonseca, F. J.; Andrade, A. M.; In: 43rd IUPAC World Polymer Congress - Polymer Science in the Service of Society, Glasgow, Escócia, 2010.

23. Santos, E. R.; Burini, E. C.; Wang, S. H.; Ozone Sci. Eng. 2012, 34, 129.

24. Zhong, Z. Y.; J. Electron. Spectrosc. Relat. Phenom. 2007, 160, 29.

25. Guerra, E. V.; Sonnenberg, V.; Junior, E. C. B.; Wang, S. H.; Santos, E. R.; Boletim Técnico da Faculdade de Tecnologia de São Paulo, 2010, $12,120$.

26. Guerra, E. V.; Sonnenberg, V.; Santos, G.; Fonseca, F. J.; Andrade, A. M.; Junior, E. C. B.; Wang, S. H.; Santos, E. R.; Boletim Técnico da Faculdade de Tecnologia de São Paulo, 2010, 12, 150.

27. Moraes, J. I. B.; Junior, E. C. B.; Wang, S. H.; Onmori, R. K.; Sonnenberg, V.; Santos, E. R.; Boletim Técnico da Faculdade de Tecnologia de São Paulo, 2012, 14, 47.

28. Yoshida, S.; Trabalho de Conclusão de Curso, Faculdade de Tecnologia de São Paulo, Brasil, 2012

29. Low, B. L.; Zhu F. R.; Zhang, K. R.; Chua, S. J.; Thin Solid Films 2002 , $417,116$.

30. Nardes, A. M.; Tese de Doutorado, Universidade de Tecnologia de Eindhoven, Holanda, 2007.

31. Lim, M. Y.; Yunus, W. M. M.; Talib, Z. A.; Kassim, A.; Journal of Engineering and Technology Research 2011, 3, 171.

32. Djobo, S. O; Bernède, J. C.; Kossi, N.; J. Mater. Sci. - Mater. Electron. 2004, 15, 579 .

33. Kido, J.; Shionoya, H.; Nagai, K. Nagai; Appl. Phys. Lett. 1995, 67, 2281.

34. Kim, D. H.; Lim, K.G.; Park; J. H.; Lee, T. W.; ChemSusChem 2012, 5 , 2053.

35. Guerra, E. V.; Estudo do desempenho de dispositivos diodos poliméricos-orgânicos emissores de luz utilizando-se camada PEDOT:PSS, Trabalho de Conclusão de Curso, Faculdade de Tecnologia de São Paulo, Brasil, 2011.

36. Khalifa, M. B.; Vaufrey, D.; Bouazizi, A.; Tardy, J.; Maaref, H.; Mater. Sci. Eng., C 2002, 21, 277. 